\title{
Lumbosacropelvic junction reconstruction resulting in early ambulation for patients with lumbosacral neoplasms or osteomyelitis
}

\author{
Stefan A. Mindea, M.D., Sean A. Salehi, M.D., Aruna Ganju, M.D., \\ Michael K. Rosner, M.D., Brian A. O’Shaughnessy, M.D., Allan Jorge, M.D., \\ AND STEPHEN L. ONDRA, M.D.
}

Department of Neurological Surgery, The Feinberg School of Medicine and McGaw Medical Center, Northwestern University, Chicago, Illinois

\begin{abstract}
Lumbosacropelvic junction instability may result from a variety of disease processes including primary and metastatic sacral tumors and degenerative disease. Regardless of the origin of the disease, restoring or maintaining spinal stability at this junction is essential for normal translation of axial forces from the lumbar spine and sacrum to the pelvis. Spinal stability is also critical for maintaining structural integrity, preventing neurological function deterioration, and alleviating resultant mechanical or axial pain. In this report, the authors describe one option for safe and effective spinal pelvic stabilization by using a transiliac rod and iliac bolt construct, which results in early postoperative ambulation, preserved neurological function, and reduced axial pain in selected patients.
\end{abstract}

Key WoRdS • spinal fixation • metastasis • lumbosacral • metastasis • tumor

Lumbosacropelvic decompression and reconstruction are surgical interventions frequently performed in patients with neoplastic or degenerative disease processes that impair axial load transfer to the pelvis. Traditionally, surgical treatment for these disease processes was often associated with prolonged immobilization during the recovery phase and significant neurological compromise stemming from neural element sacrifice during surgery. ${ }^{5,6,10,12,15,17,19,21}$ The QOL these patients experienced postoperatively was quite poor and especially disheartening was that for patients with a limited life expectancy.

We present a report of six patients treated with epidural decompression and lumbosacropelvic reconstruction using a surgical technique that provides immediate lumbosacral stability translating into substantially reduced postoperative immobilization with no additional compromise of neurological function.

\section{CLINICAL MATERIAL AND METHODS}

From January 1, 1998, to January 1, 2002, six patients treated by the senior author (S.L.O.) required spinopelvic

Abbreviations used in this paper: $\mathrm{MR}=$ magnetic resonance; $\mathrm{QOL}=$ quality of life. stabilization and reconstruction for metastatic sacral neoplasms (four patients), primary sacral neoplasms (one patient), and severe osteomyelitis (one patient). The four patients reported in this series with metastatic sacral tumors had the following diagnoses (Table 1): metastatic adenocarcinoma of the lung in two, metastatic paraganglioma in one, and metastatic thoracic fibrosarcoma in one. Primary giant cell tumor of the sacrum was diagnosed in one. Two patients had undergone prior pelvic radiation therapy: one for recurrent metastasis to the sacrum (Case 3) and the other for uterine cancer (Case 6). One patient (Case 3) had also undergone previous lumbosacral stabilization at another institution before presenting to our medical center. The mean patient age was 44 years; there were five men and one woman. Preoperative symptoms included lowback pain, radiculopathy, bladder incontinence, lower-extremity weakness, and saddle block paresthesia. Five of the patients were ambulatory with or without assistance preoperatively; one patient (Case 6) was bedridden.

Preoperative computerized tomography and MR imaging of the lumbosacral spine and pelvis was performed in all patients. In those patients with tumor as the pathological process, most of the tumor burden was focused in the sacrum (Fig. 1). Tumor extension was present at the S-1 endplate and extended to at least the midsacrum in all patients. The L-5 vertebral body was infiltrated by tumor in 
TABLE 1

Synopsis of pre-and postoperative symptoms and motor examinations in six patients*

\begin{tabular}{|c|c|c|c|c|c|c|}
\hline \multirow{2}{*}{$\begin{array}{l}\text { Case } \\
\text { No. }\end{array}$} & \multirow{2}{*}{$\begin{array}{l}\text { Age } \\
\text { (yrs) }\end{array}$} & \multirow[b]{2}{*}{ Diagnosis } & \multicolumn{2}{|c|}{ Preop } & \multicolumn{2}{|l|}{ Postop } \\
\hline & & & Symptoms & Strength & Symptoms & Strength \\
\hline 1 & 56 & $\begin{array}{l}\text { metastatic fibro- } \\
\text { sarcoma }\end{array}$ & $\begin{array}{l}\text { LBP, S-1 radiculopathy, } \\
\text { urinary incontinence }\end{array}$ & $5 / 5$ all extremities & $\begin{array}{l}\text { LBP, radiculopathy \& bladder } \\
\text { symptoms resolved }\end{array}$ & $5 / 5$ all extremities \\
\hline 2 & 52 & $\begin{array}{l}\text { metastatic lung } \\
\text { cancer }\end{array}$ & $\begin{array}{l}\text { LBP, rt S-1 radiculopathy, } \\
\text { rt foot weakness }\end{array}$ & rt 3/5 plantar flexion & none & $\begin{array}{l}\text { rt } 4 / 5 \text { plantar flex- } \\
\text { ion }\end{array}$ \\
\hline 3 & 35 & $\begin{array}{l}\text { metastatic para- } \\
\text { ganglioma }\end{array}$ & $\begin{array}{l}\text { LBP, L-5 radiculopathy, } \\
\text { urinary incontinence, } \\
\text { saddle anesthesia }\end{array}$ & bilat $4 / 5$ dorsiflexion & $\begin{array}{l}\text { bilat foot weakness, resolved } \\
\text { bladder incontinence }\end{array}$ & $\begin{array}{l}\text { bilat } 3 / 5 \text { dorsiflex- } \\
\text { ion }\end{array}$ \\
\hline 4 & 46 & $\begin{array}{l}\text { metastatic lung } \\
\text { cancer }\end{array}$ & $\begin{array}{l}\text { LBP, lt S-1 \& S-2 radicu- } \\
\text { lopathy, urinary retention }\end{array}$ & lt $4 / 5$ plantar flexion & $\begin{array}{l}\text { normal bladder function, } \\
\text { transient 1t L-5 radiculop- } \\
\text { athy }\end{array}$ & lt $4 / 5$ dorsiflexion \\
\hline 5 & 22 & $\begin{array}{l}\text { primary giant cell } \\
\text { tumor }\end{array}$ & $\begin{array}{l}\text { LBP, rt buttock pain w/ } \\
\text { bilat S-1 radiculopathy }\end{array}$ & $5 / 5$ all extremities & none & $5 / 5$ all extremities \\
\hline 6 & 51 & sacral osteomyelitis & $\begin{array}{l}\text { LBP, rt L-5 \& S-1 radicu- } \\
\text { lopathy }\end{array}$ & $\begin{array}{l}\text { diffuse rt leg weakness } \\
\text { (range } 2 / 5-3 / 5)\end{array}$ & $\begin{array}{l}\text { radiculopathy resolved, } \\
\text { transient pelvic \& LBP }\end{array}$ & $\begin{array}{l}\text { bilat } 4 / 5 \text { plantar } \& \\
\text { dorsiflexion }\end{array}$ \\
\hline
\end{tabular}

* LBP = low-back pain.

one patient (Case 3). Severe bone destruction was seen in all cases. At initial presentation to our medical center, all patients with sacral tumors demonstrated a sacroiliac joint devoid of any tumor involvement. Preoperative angiography was performed in all patients with sacral tumor, with two patients (Cases 3 and 5) requiring embolization because of extensive tumor vascularity.

\section{Surgical Technique}

Three (Cases 1, 2, and 4) of the four patients who did not require preoperative embolization, based on angiography, underwent anterior and posterior column decompression and reconstruction exclusively via a posterior approach. Surgery in the fourth patient (Case 6) was converted from an initial posterior approach to a posterioranterior approach after the intraoperative dissection was severely compromised by extensive fibrosis, presumably from the patient's history of radiation to the pelvis for treatment of uterine cancer.
Of the two patients (Cases 3 and 5) who required preoperative embolization, one patient was treated via a posterior approach with a nerve-sparing technique and the other was treated via an anterior-posterior approach; there was an 8-day interval between approaches.

Epidural decompression and tumor resection was performed via the approaches outlined previously. In the majority of patients, a single-stage posterior approach was sufficient for decompression and reconstruction. Typically, a posterior midline incision from L-3 to the sacrum would be made followed by exposure of the posterior elements and laminectomy at the L-5 level. Occasionally, an L-4 laminectomy would also be performed if significant L-5-associated disease was present. In addition, if the tumor significantly compromised the S-1 endplate, a bilateral facetectomy would be performed. Next, the entire sacral canal was undomed laterally to the border with the pedicles. The lumbosacral nerve roots, thecal sac, and tumor were identified, and the tumor was dissected free of

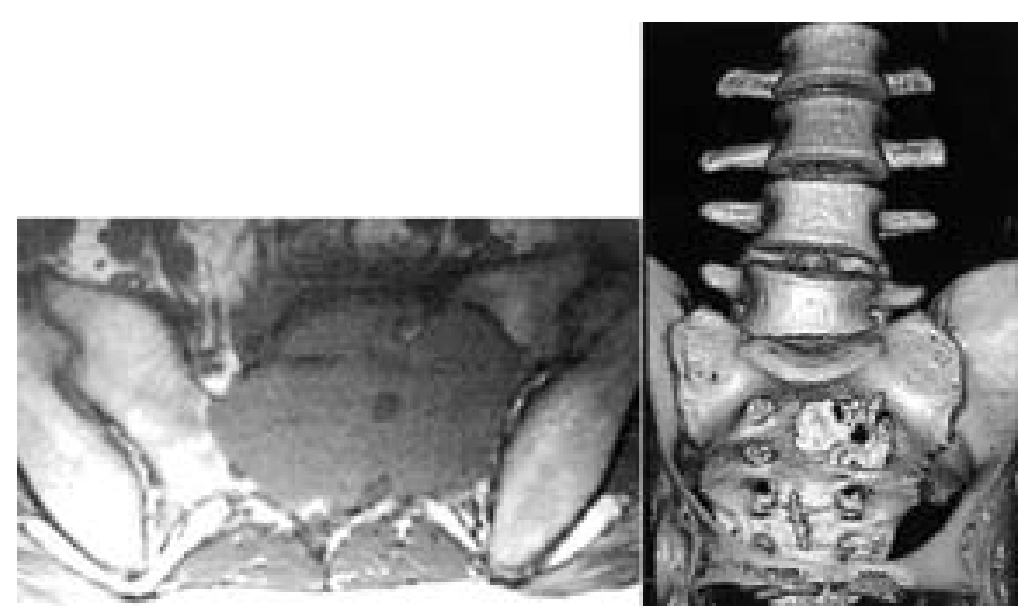

Fig. 1. Left: An MR image of sacrum at the level of S-2 obtained in the patient with fibrosarcoma metastasis to the sacrum. The majority of the sacrum is involved with tumor. Right: A three-dimensional reformatted picture of the lumbosacral region. Note the punched-out lesion at S-2. 
the thecal sac by using an ultrasonic aspirator (CUSA; Valleylab, Boulder, CO) in conjunction with standard microscopic dissection techniques. Once this dissection was complete, each nerve root was visualized and tracked as it entered the tumor or anterior wall of the sacrum. Again, the tumor was dissected free of the nerve root by using the aspirator and microscopic dissection techniques; additional exposure could be achieved by retracting the thecal sac and nerve roots, thus creating appropriate access for the aspirator to function between the nerve roots. Somatosensory evoked potentials and intraoperative electromyography for nerve root monitoring were routinely performed. Complete resection of the tumor and diseaseinfiltrated bone was accomplished down to the anterior pelvic fascia. The lateral resection of the sacrum may extend out but not through the sacroiliac joints if reconstruction that allows mobilization is to be considered. ${ }^{11}$

In reconstruction of the anterior column, the technique used in our patients was grounded in achieving two biomechanical goals: providing axial support to the spine and load transfer to the pelvis. To attain these goals, we introduced a titanium mesh cage filled with bone allograft between the retracted nerve roots against the endplate of L-5 (Fig. 2). The cage had a hole that allowed for the eventual passage of a 0.25 -in titanium rod, which served to bridge the axial load onto the ilium bilaterally. A Kirschner wire, which serves as a guidewire for the passing of a reamer and titanium rod through the cage, was passed using fluoroscopic guidance through the titanium cage and contralateral ilium and hip. The construct is such that the Kirschner wire stems through both iliacs via the hole in the titanium cage. Next, a 0.25 -in cannulated reamer was passed over the Kirschner wire, perforating the ilium bilaterally. Finally, once the path along the Kirschner wire had been dissected free by the cannulated reamer, the 0.25-in titanium bar was advanced over the Kirschner wire through the cage and ilium bilaterally. To prevent movement and shifting of the rod, connectors were placed at the ends of the bar that fastened against the ilium bilaterally. The cage was stabilized in part by the broad contact it had with the L-5 endplate as well as by the teeth in the cage surface, which were compressed into the L-5 vertebral body by axial load forces.

Having provided axial support and load transfer to the pelvis, we then turned our attention to reconstruction of the posterior tension band when indicated. Of note, posterior tension band reconstruction may be deferred in selected instances, most notably if the facet joints and pars interarticularis are preserved. Several options exist for this reconstruction. Typically, we proceeded with segmental pedicle screw fixation at L-4 and L-5 with attachment of the screws to the ilium by means of a modified Galveston technique $^{1}$ (Figs. 3 and 4).

\section{RESULTS}

A comparison of the preoperative and 1-month postoperative findings is presented in Table 1. Low-back pain, a symptom in all patients, was significantly improved in five of six patients and was reduced to transient pain in another. With regard to motor function, one patient incurred a new neurological deficit, three patients experi-

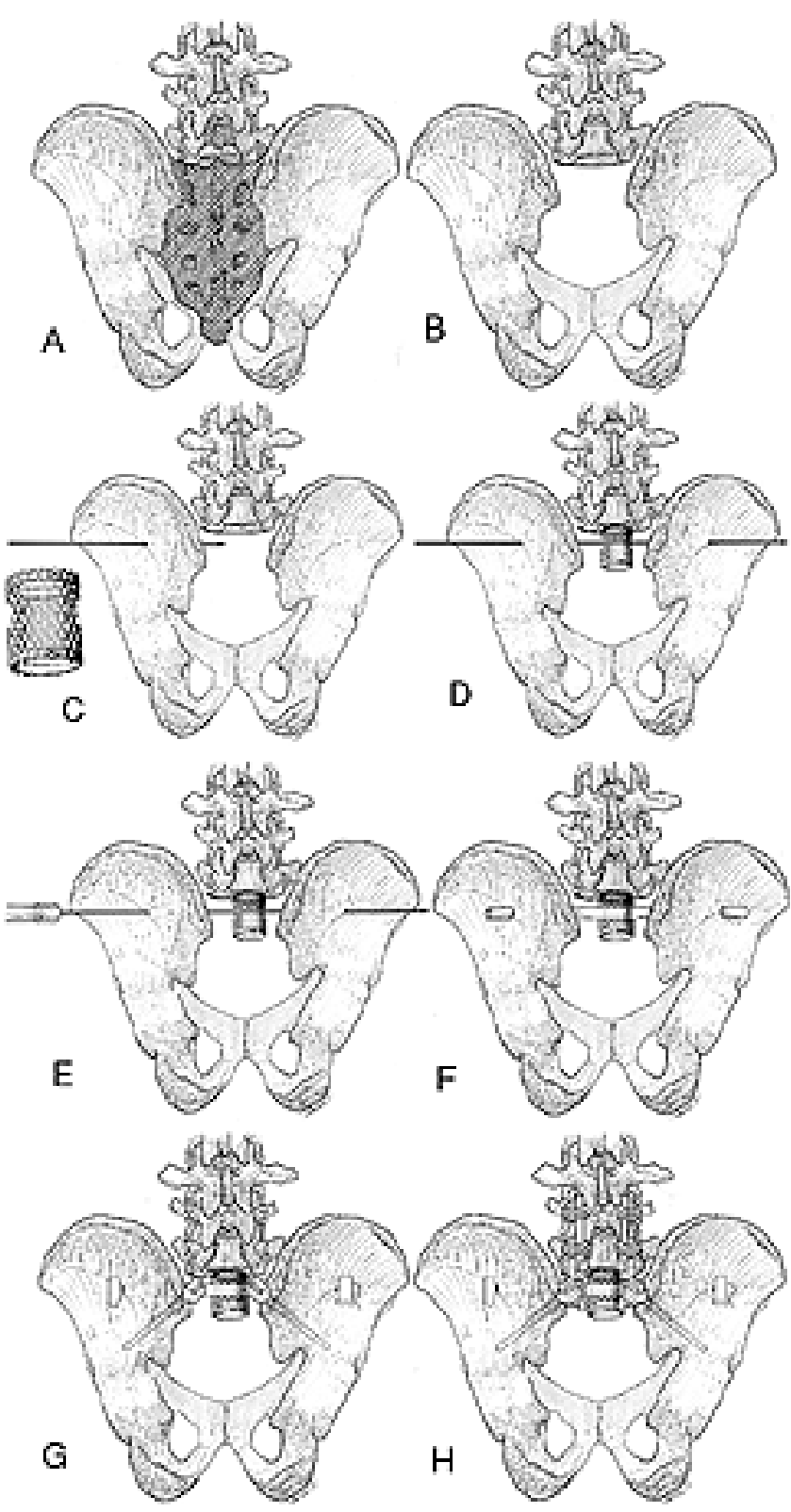

Fig. 2. Artist's illustrations of the stepwise reconstruction of the lumbosacral junction. A: Dorsal view of the lumbosacropelvic junction. The hatched area will be resected. B: The sacrectomy is complete. C: A K-wire passes through the left ilium in the resected region of S-1. D: A K-wire passes through the cage and the right ilium. E: The reamer is passed over the guidewire. F: A 0.25-in titanium rod passes through the left ilium, the cage, and the right ilium. G: Lumbar pedicle screws, Farsi ilial screws, and the side-tighting bolts at either end of ilial rod are placed. H: Pedicle screws are attached to the ilial screws by using rods. This is the final stage of lumbopelvic fixation.

enced no new deficits, and two patients actually demonstrated an improvement in motor capacity. Bladder function also was normalized in the three patients with preoperative urinary incontinence or retention and no patient incurred any new bowel or bladder impairment.

All patients underwent intense physical and occupational therapy on being transferred to the neurosurgical 


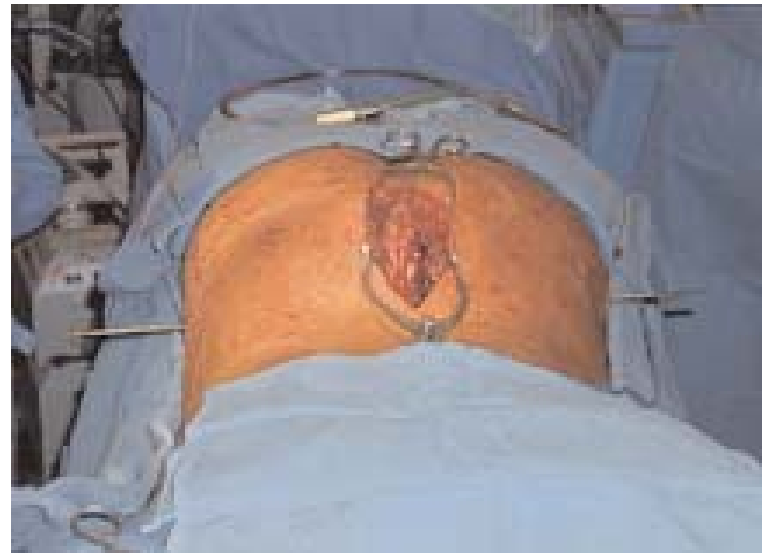

Fig. 3. Intraoperative photograph of the transilial rod first traversing the right ilium, passing through the cage, and then through the left ilium.

floor. The level of activity was increased gradually and all patients participated in physical rehabilitation exercises while wearing rigid external thoracolumbosacral orthoses. On discharge, all patients were walking with or without assistance between postoperative Days 6 and 14 (mean 10 days). Four patients were discharged home ambulating independently; two patients were ambulating with assistance.

There was no surgery-related death. Nonneurological complications included internal iliac vein thrombosis (one patient), pulmonary embolus requiring Greenfield filter placement (one patient), pneumonia (one patient), and disseminated intravascular coagulopathy (one patient).

In the follow-up period, instrumentation revision was required in two patients (Cases 1 and 6) at 6 and 16 weeks after surgery, respectively. In the patient in Case 1, the transiliac rod was found to be loosened and rod repositioning with new locking screw placement was performed. Three weeks later ( 9 weeks after the initial surgery), the patient returned complaining of leg weakness and lowback pain. Follow-up lumbosacral MR imaging demonstrated tumor recurrence with extension into the sacroiliac joint and the patient underwent repeated tumor debulking and decompression with revision of one iliac bolt. The patient maintained his capacity to walk until he died 9 months postoperatively. The patient in Case 6 also underwent instrumentation revision for failure of both iliac screws. She continues to walk without assistance and is without any neurological complication.

One patient (Case 2) died 15 months postoperatively of pulmonary and liver metastases but continued to walk until the time of his medical deterioration. Another patient (Case 3) continues to walk 14 months postoperatively; however, he has not returned for follow-up care since that time. One patient (Case 4) died 7 months postoperatively of pulmonary adenocarcinoma but continued to walk until her medical deterioration ensued. One patient (Case 5) continues to walk 31 months postoperatively and is without neurological deficits. He has since undergone local radiation therapy because of the subtotal resection we performed and the high recurrence rates associated with this tumor.
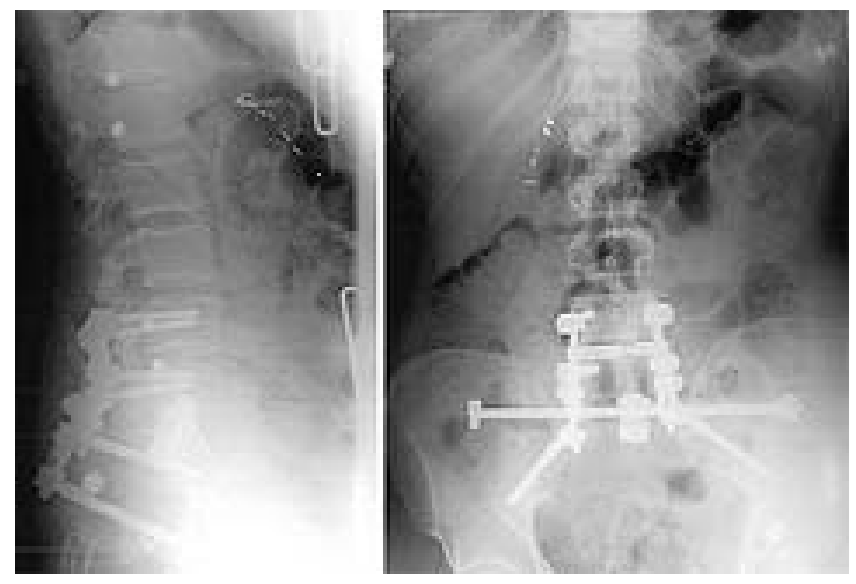

Fig. 4. Anteroposterior (left) and lateral (right) radiographs obtained after a lumbosacral reconstruction.

\section{DISCUSSION}

Patients in whom metastatic or primary sacral tumors are diagnosed as well as patients with severe degenerative disease in the sacrum may experience instability at the lumbopelvic junction. Instability in this region of the spinal column frequently accounts for the majority of the symptoms these patients experience. Traditionally, reconstruction of the lumbopelvic junction succeeded in stabilizing the spine from a biomechanical standpoint; however, the benefit in patient functionality was often deferred for several months. Immobilization of 3 to 6 months' duration along with new postsurgical neurological deficits are commonly reported in the literature..$^{5,6,10,12,15,17,19,21}$ In this article, we demonstrate that patients with lumbosacral instability may undergo reconstructive surgery that incurs fewer neurological deficits and earlier mobilization compared with more traditional methods.

\section{Spinal Instability}

When assessing instability in the lumbosacropelvic region, one must consider the anterior column, axial load transmission to the pelvis, the posterior tension band, and pelvic ring competency. Although all of these factors are essential to biomechanical stability, stability is predicated on a strong anterior column. The absence of a strong anterior column results in increased stress placed on the posterior elements via cantilever forces, impairment of normal axial load transfer, and up to a $50 \%$ reduction in pelvic strength. ${ }^{7}$

As previously mentioned, axial load transfer is impaired if the integrity of the anterior column is compromised secondary to tumor involvement, resection, or other degenerative processes. Because the anterior column bears approximately $80 \%$ of the axial load, structural instability in this column significantly alters normal weight bearing. The anterior column may be deemed unstable if the disease process extends to $1 \mathrm{~cm}$ below the sacral promontory and extends to more than two thirds of the sacral endplate. ${ }^{7}$ In this instance, repair of the anterior column is mandated to restore axial load transmission from the spinal column to the pelvis; however, at locations other than $\mathrm{S}-1$, reconstruction of a vertebrectomy defect alone, 
in selected cases, may provide satisfactory support without the use of additional fixation. ${ }^{4}$ Using human cadaveric specimens, Kanayama, et al., ${ }^{9}$ recently reported that anterior reconstruction provided stiffness equivalent to circumferential reconstruction provided that only corpectomy or subtotal spondylectomy had been performed.

Not infrequently, the posterior tension band may be weakened by tumor invasion into the posterior elements or by increased cantilever forces secondary to a significant anterior column defect. Therapeutic intervention such as laminectomy as well as infective or degenerative disease processes may also decrease bone density and quality, further stressing the posterior tension band. Because only $20 \%$ of the axial load is carried by the posterior tension band, it may be reasonable to defer reconstruction if the middle column, along with the anterior column, remains structurally sound. ${ }^{11}$ In instances in which there is substantial anterior column compromise, the senior author recommends that the posterior tension band be repaired with segmental instrumentation to relieve any additional stress placed on posterior elements by the shift in axial load. We do, however, recognize that the three-column concept described by Denis ${ }^{3}$ was developed to evaluate thoracolumbar fractures and, as such, may not always be applicable in cases involving neoplastic destruction of the spine because of the differing mechanisms resulting in spinal instability. ${ }^{4}$ Bearing in mind the biomechanical concepts, surgical management of these patients is quite complicated and a perfect algorithm for treatment in all cases is currently nonexistent.

Pelvic ring competency must be ensured for a stable lumbopelvic construct. The pelvic ring may be restored by a transiliac bar in cases in which the sacrum is removed. Pelvic ring competency is vital to the transfer of axial load onto the pelvis and then onto the femurs.

\section{Surgical Goals}

Taking into account the aforementioned factors, stabilization and reconstruction of the lumbopelvic junction are performed with two distinct surgical goals: restoration of the pelvic ring and axial load transfer from the spinal column to the pelvis. Surgical objectives with regard to the treatment of the primary disease process include maximal decompression and tumor debulking for metastatic sacral tumors and maximal debridement of infected bone in osteomyelitis. In cases of primary sacral tumors, an en bloc resection with neural element sacrifice is frequently performed to ensure maximal tumor extirpation. ${ }^{2,8,13,20}$ In our patient with primary giant cell tumor, en bloc resection was deferred because of the relatively young age of the patient as well as significant morbidity associated with this method. In hopes of maximizing neural element preservation, the patient elected to undergo posterior grosstotal resection with a nerve-sparing technique.

\section{Outcome Goals}

Immediate spine stability is necessary to achieve early ambulation in patients requiring lumbosacropelvic reconstruction. In patients with metastatic sacral tumors, surgical intervention is strictly palliative and the focus of care shifts to one of preserving QOL. Recovery times of 3 to 6 months in patients who undergo traditional stabilization methods may often not be feasible for this subset of patients. The authors believe that early ambulation, pain control, and preservation of neurological function are all realistic goals in these patients. ${ }^{11,14}$ A comprehensive technique grounded in providing immediate spinal stabilization may serve to maximize the QOL in these patients.

\section{Surgical Approaches}

The initial treatment evaluation of sacral tumors must take into account the following tumor characteristics: tumor vascularity, encroachment of the anterior pelvic fascia, and the tumor location relative to the S-1 level. Lesions below $\mathrm{S}-1$ are considered caudal and those at the S-1 level are considered proximal. This distinction is significant because the treatment modalities and subsequent approaches for each are vastly different. In general, caudal lesions are structurally stable lesions and seldom require reconstruction. Proximal lesions, on the other hand, may alter the biomechanics at the lumbosacral junction substantially and may therefore more frequently require stabilization. $2,13,15,20$

The indications for a combined anterior-posterior approach include the following circumstances: extensive tumor vascularity, primary proximal sacral tumor engaging the lumbosacral junction in an extensive fashion (in particular the S-1 endplate), and disease that penetrates the anterior pelvic fascia. Proceeding with an anteriorposterior approach in these scenarios ensures optimal hemostasis and adequate exposure for en bloc resection when indicated. In all other circumstances, the senior author prefers a single-stage posterior approach that permits both decompression and reconstruction.

\section{Comparison With Other Surgical Techniques}

Traditional approaches for dealing with instability at the lumbosacropelvic junction secondary to various causes differ most notably with regard to providing immediate spinal stability. We advocate the use of the pyramesh cage-induced axial support with the transfer of spinal axial load via a transiliac bar and posterior segmental reconstruction in which pedicle screws and iliac bolts are used because this construct provides immediate lumbosacropelvic stabilization. ${ }^{11,14}$ Ultimately, this translates into earlier ambulation and improved QOL for the patient when compared with more traditional reconstructive strategies. 6,8,11,14,16,18,19 Although we consider the nerve-sparing technique described in this report to be appropriate for proximal metastatic sacral tumors as well as severe lumbosacral degenerative disease, we acknowledge when a gross-total resection is indicated, as is often the case with primary sacral tumors. $2,13,15,20$

\section{CONCLUSIONS}

Symptoms in patients in whom neoplastic or degenerative pathological entities involving the sacrum have been diagnosed often stem from instability between the spinal column and pelvis. Reconstruction with the goal of establishing immediate spinal stability is paramount in achieving earlier ambulation, preservation of neurological elements, and preservation of QOL, especially for those patients who have a limited life expectancy. We believe 
that the technique described in this report is a valuable addition to the treatment armamentarium in patients afflicted with these conditions because it provides immediate spine stabilization as well as reduced neurological debilitation after surgery. Ultimately, these two factors translate into an improved QOL for patients requiring lumbosacropelvic stabilization.

\section{Disclosure}

No grant or other financial assistance was provided in conjuction with this report.

\section{References}

1. Allen BL Jr, Ferguson RL: The Galveston technique for L rod instrumentation of the scoliotic spine. Spine 7:276-284, 1982

2. Barwick KW, Huvos AG, Smith J: Primary osteogenic sarcoma of the vertebral column: a clinicopathologic correlation of ten patients. Cancer 46:595-604, 1980

3. Denis F: The three column spine and its significance in the classification of acute thoracolumbar spinal injuries. Spine 8: $817-831,1983$

4. Fourney DR, Gokaslan ZL: Spinal instability and deformity due to neoplastic conditions. Neurosurg Focus 14 (1):Article 8, 2003

5. Gennari L, Azzarelli A, Quagliuolo V: A posterior approach for the excision of sacral chordoma. J Bone Joint Surg Br 69: $565-568,1987$

6. Gokaslan ZL, Romsdahl MM, Kroll SS, et al: Total sacrectomy and Galveston L-rod reconstruction for malignant neoplasms. Technical note. J Neurosurg 87:781-787, 1997

7. Gunterberg B, Romanus B, Stener B: Pelvic strength after major amputation of the sacrum. An experimental study. Acta Orthop Scand 47:635-642, 1976

8. Jackson RJ, Gokaslan ZL: Spinal-pelvic fixation in patients with lumbosacral neoplasms. J Neurosurg (Spine 1) 92: 61-70, 2000

9. Kanayama M, Ng JT, Cunningham BW, et al: Biomechanical analysis of anterior versus circumferential spinal reconstruction for various anatomic stages of tumor lesions. Spine 24: 445-450, 1999
10. Localio SA, Eng K, Ranson JH: Abdominosacral approach for retrorectal tumors. Ann Surg 191:555-560, 1980

11. Ondra SL, Salehi SA, Ganju A: Primary and metastatic disease of the sacrum and lumbar-sacral junction, in Batjer HH, Loftus CM (eds): Textbook of Neurological Surgery: Principles and Practice. Philadelphia: Lippincott Williams \& Wilkins, 2003, Vol 2 pp 1832-1838

12. Ozdemir MH, Gurkan I, Yildiz Y, et al: Surgical treatment of malignant tumours of the sacrum. Eur J Surg Oncol 25:44-49, 1999

13. Rosen G, Caparros B, Nirenberg A, et al: Ewing's sarcoma: ten-year experience with adjuvant chemotherapy. Cancer 47: 2204-2213, 1981

14. Salehi SA, McCafferty RR, Karahalios D, et al: Neural function preservation and early mobilization after resection of metastatic sacral tumors and lumbosacropelvic junction reconstruction. J Neurosurg (Spine 1) 97:88-93, 2002

15. Samson IR, Springfield DS, Suit HD, et al: Operative treatment of sacrococcygeal chordoma. A review of twenty-one cases. J Bone Joint Surg Am 75:1476-1484, 1993

16. Shikata J, Yamamuro T, Kotoura Y, et al: Total sacrectomy and reconstruction for primary tumors. Report of two cases. J Bone Joint Surg Am 70:122-125, 1988

17. Stener B, Gunterberg B: High amputation of the sacrum for extirpation of tumors. Principles and technique. Spine 3:351-366, 1978

18. Sung HW, Shu WP, Wang HM, et al: Surgical treatment of primary tumors of the sacrum. Clin Orthop 215:91-98, 1987

19. Tomita K, Tsuchiya H: Total sacrectomy and reconstruction for huge sacral tumors. Spine 15:1223-1227, 1990

20. Weinstein JN, McLain RF: Primary tumors of the spine. Spine 12:843-851, 1987

21. Wuisman P, Harle A, Matthiass HH, et al: Two-stage therapy in the treatment of sacral tumors. Arch Orthop Trauma Surg 108:255-260, 1989

Manuscript received June 23,2003

Accepted in final form July 3, 2003.

Address reprint requests to: Stephen L. Ondra, M.D., Department of Neurological Surgery, Northwestern University, 233 E. Erie Street, Suite 614, Chicago, Illinois 60611. email: sondra@ nmff.org. 